\title{
Conflation of satellite altimetry and tide gauge records at coast
}

DOI: https://doi.org/10.1515/jogs-2020-0113

Received February 27, 2020; accepted May 28, 2020

\begin{abstract}
This study demonstrates that absolute (geocentric) and relative sea level trends, sea level acceleration, low frequency sea level variations and linear trends in vertical crustal movements experienced at a tide gauge station can be estimated simultaneously using conflated satellite altimetry and tide gauge measurements without the aid of GPS measurements. The formulation is the first of its kind in sea level studies and its effectiveness is exemplified using tide gauge, and satellite altimetry measurements carried out in the vicinity of a tide gauge station.
\end{abstract}

Keywords: Climate change; Sea level rise; Satellite altimetry; Tide gauge; Sea level trend and acceleration; Vertical crustal movements

\section{Introduction}

Tide gauge, TG, and satellite altimetry, SA, measurements are primary technologies for monitoring, analyzing and modelling sea level variations. While SA measurements are intrinsically absolute, i.e. geocentric, TG measurements are relative as they are referenced to the earth's crust that may be affected by vertical crustal movements, VCM. Today SA measurements are ubiquitous, yet they span only 24 years. However, although TG stations are limited in number globally, and sample only coastal areas, TG records can be century long thereby better suited for detecting and quantifying relative sea level trends and low frequency sea level variations that confound detecting and estimating sea level trends and accelerations using SA measurements. The best of the two worlds is therefore their conflation. To achieve this end, one can estimate relative sea level trend and acceleration using TG measurements and correct the estimated relative trend using global positioning system, GPS, measurements for the effect of the VCM. Currently, this approach is limited due to lack

\footnotetext{
*Corresponding Author: H. Bâki Iz: Division of Geodetic Science, School of Earth Sciences, The Ohio State University Columbus, Ohio, USA, E-mail: H.Baki.Iz@gmail.com

C. K. Shum, T. Y. Yang: Division of Geodetic Science, School of Earth Sciences, The Ohio State University Columbus, Ohio, USA
}

of sufficiently long GPS measurements at TG stations or in their vicinities. However, SA measurements are geocentric, and their time span are longer than the GPS measurements, thereby they can be used to provide information about VCM.

The use of TG and SA measurements and geological data to estimate VCM is not a new idea. Fadil et al., (2013) offers an exhaustive review of studies in this area. More recently, Iz, et al., (2017), and Iz et al. (2019) introduced and demonstrated optimal mathematical and statistical methods to achieve this end. This study, however, differs from the previous ones in terms of both mathematical and statistical models, which enable simultaneous estimation of absolute and relative sea level trends and acceleration, low frequency sea level variations and VCM experienced at a TG station using conflated SA and TG measurements without the aid of geological information or GPS measurements.

In the following sections, first, we describe monthly SA altimetry records nearby a TG station, namely, Atlantic City, USA used in this study. We then estimate relative sea level trend and acceleration using monthly TG data, and absolute sea level trend and acceleration using SA data. The difference between the estimated relative and absolute sea level trends gives the linear rate of VCM experienced at the TG station. These results are used as baseline estimates for the subsequent solution. In what follows, we estimate all the aforementioned model parameters simultaneously using a conflation model with restricted Least Squares for a comparison. The estimated VCM of the conflation model is then contrasted against the VCM obtained using GPS measurements carried out in the vicinity of the tide gauge station for verification.

\section{Atlantic City, USA, Tide Gauge and Satellite Altimetry Data}

Atlantic City, USA, TG station was selected for the numerical demonstration of the models to be discussed in the following sections. The TG measurements at this location exhibits various low frequency sea level variations and also 
experience significant VCM as revealed by GPS measurements at this locality (16 km away from the TG station).

TG time series were downloaded from the Permanent Mean Sea Level (PSMSL) repository (PSMSL, 2018) as shown in Figure 1. TG time series are referenced to the Revised Local Reference (RLR). Because no corrections for the post glacial rebound (PGR) or local vertical movements were applied to the data, they represent relative sea level changes with respect to the Earth's crust.

Although the SA measurements are already corrected for the effect of atmospheric pressure, TG measurements are not. In this study, ERA-Interim (Dee et al., 2011) and NOAA-CIRES 20th Century Reanalysis V2c (Compo et al., 2006) monthly averaged surface pressure product were used for computing inverted barometer, IB, correction. The ERA-Interim dataset for the Key West TG station was downloaded from European Centre for Medium-Range Weather Forecasts archive (ECMWF, 2019, Berrisford et al., 2011). The data reflect the monthly averaged surface pressure on a regular $0.75^{\circ} \times 0.75^{\circ}$ longitude/latitude grid for the period from Jan 1979 to Oct 2018. Because ERA-Interim does not provide the data before 1979, we downloaded NOAA-CIRES Reanalysis surface pressure dataset as well from NOAA ESRL Physical Sciences Division archive. The monthly mean surface pressure data for Jan 1851 to Dec 2014 are presented by $2^{\circ} \times 2^{\circ}$ longitude/latitude grid. The mean of differences between ERA-Interim and NOAA Reanalysis are computed using overlapping period of two dataset at each station, and then added to the ERA-Interim data. TG measurements shown in Figure 1 are corrected for the IB effect.

The sea level around the Atlantic City, USA, TG station was also surveyed by various SA for geocentric mean sea level, MSL, variations/anomalies. We used SA sea level anomaly data produced by the NASA's MEaSURe's program, which were downloaded on January 2019 for the region $24^{\circ} \mathrm{N}, 82^{\circ} \mathrm{W}-25^{\circ} \mathrm{N}, 81^{\circ} \mathrm{W}$ for the period Dec 1992 - Jun 2017 (Figure 1). The time series includes data from TOPEX/Poseidon (T/P), Jason-1, Ocean Surface Topography Mission/Jason-2 (OSTM) and Jason-3 primary mission series (TPJAOS) (Zlotnicki et al., 2016).

\section{Generic kinematic and statistical trend models for satellite altimetry and tide gauge records}

Iz (2014, 2015) developed a kinematic model consisting of a secular trend, acceleration, and statistically signif-

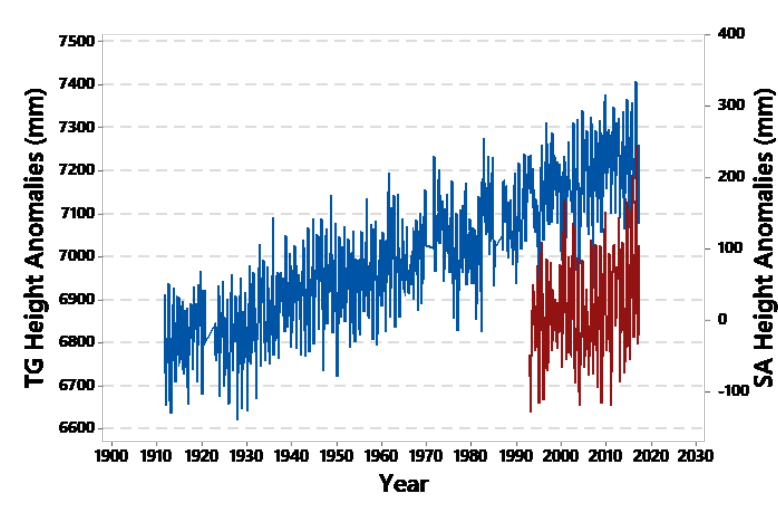

Fig. 1. Overlapping Atlantic City TG (in blue) and SA monthly sea level measurements used in this study. Each time series refers to its own datum.

icant and globally prevalent specific low frequency sea level variations that are due to the coupling of astronomical forcing of luni-solar origin, in tandem with other natural broadband internal ocean-atmosphere interactions, including atmospheric and thermosteric contributions. Their interaction produces signatures at multi-decadal time scales known as sub and super harmonics (ibid.) shown in Figure 2.

With this a priori understanding about the underlying sea level variations, the kinematic and statistical model for both SA and TG time series reads as,

$$
\begin{aligned}
h_{t}= & h_{t_{0}}+v\left(t-t_{0}\right)+\frac{a}{2}\left(t-t_{0}\right)^{2} \\
& +\sum_{k=0}^{m}\left\{\alpha_{k} \sin \left[\left(\frac{2 \pi}{P_{k}}\right)\left(t-t_{0}\right)\right]\right. \\
& \left.+y_{k} \cos \left[\left(\frac{2 \pi}{P_{k}}\right)\left(t-t_{0}\right)\right]\right\}+\varepsilon_{t}
\end{aligned}
$$

In this model, monthly averaged tide gauge data, $h_{t}$, are observed at $t=t_{\text {Start }} \ldots t_{E n d}$. The epochs of the measurements are shifted to the middle of the series, $t_{0}$ for shorter time offsets to improve the numerical stability of the solution. The unknown sea level reference height $h_{t_{0}}$ is defined at the middle epoch of the measurements. The initial velocity is $v$, and $a$ is the acceleration or deceleration in sea level. The initial velocity is a relative trend if the measurements were conducted at TG stations, or geocentric (or absolute) if SA measurements are under consideration. Therefore, the above formulation is valid for both TG and SA time series with a different realization of the underlying sea level trend.

The unknown parameters $\alpha_{k}$ an $\mathrm{d} y_{k}$ are the harmonic components to be estimated and used to calculate the amplitudes and the phase angles of the periodicities stated 
above. The summation is carried over for all the harmonics $m$, listed in Figure 2. In addition, a study by Iz (2015) detected and quantified that tide gauge stations located at the West Atlantic coast were also affected by a multitude of periodic changes at decadal scales including a statistically significant $(p<0.05)$ periodicity within the 12-14 yr. range that can be attributed to wind driven currents and atmospheric pressure variations. This effect is also incorporated into the model given by eq (1).

The random variable $\varepsilon_{t}$ represents the lump-sum effect of an underlying first order autoregressive, AR(1) process, and instrument errors or other random effects in sea level changes, i.e., disturbances $u_{t}$. The autoregressive error $\varepsilon_{t}$ is expressed as,

$$
\varepsilon_{t}=\rho \varepsilon_{t-1}+u_{t} \quad t=\ldots,-2,-1,0,1,2, \ldots
$$

In this expression, $-1 \leq \rho \leq 1$ is the unknown autocorrelation coefficient of AR(1) process. Furthermore, the stochastic process for the random noise $u_{t}$, has the following assumed distributional properties,

$$
\begin{aligned}
& E\left(u_{t}\right)=0 \quad E\left(u_{t}^{2}\right)=\sigma_{u}^{2} \quad E\left(u_{t} u_{t-1}\right)=0 \\
& \left(t=t^{\prime}\right) \rightarrow \Sigma_{u}=\text { diagonal }\left(\sigma_{u}^{2}\right)
\end{aligned}
$$

The properties of the random variable $u_{t}$ in eq. (3) can be abbreviated as independent and identically distributed random variable, $u_{t} \sim i i d\left(0, \sigma_{u}^{2}\right)$, where $\sigma_{u}^{2}$ is the variance of the random variable.

In the following section, we will first describe the sea level data collected at a TG station namely, Atlantic City, USA and the SA data in its vicinity. Two sets of model parameters are then estimated independently using data from each technology for the same area.

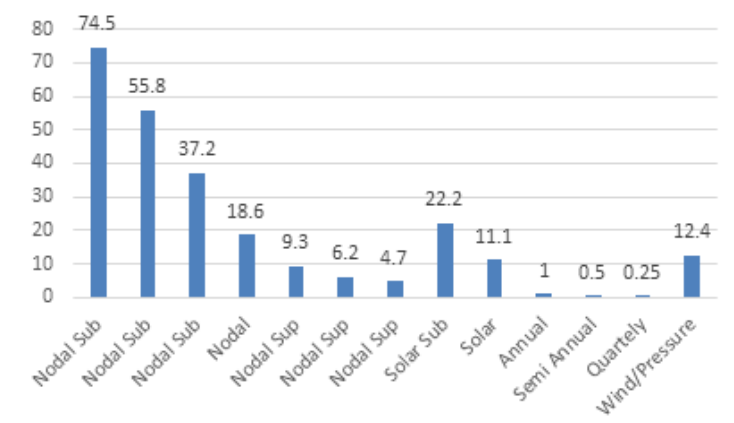

Fig. 2. Low frequency sea level variations experienced at globally distributed TG stations (Iz, 2014).

\subsection{Single Model Solutions for TG and SA at the Atlantic City}

We used the model described by eq. (1) - eq. (3) to estimate the trends for both SA and TG time series using Hildreth$\mathrm{Lu}$ procedure (Hildreth and $\mathrm{Lu}, 1960$ ), which accounts for the effect of $\mathrm{AR}(1)$ disturbances. The estimated absolute and relative sea level trends and their uncertainties, i.e. standard errors, SE, are shown in Table 1. Both sea level trends and acceleration are statistically significant at $95 \%$ confidence level, $\mathrm{CL}^{1}$. Some of the parameters are either not estimable because of the high variance inflation factors, VIF², leading to harmful collinearity, or they were not statistically significant. The amplitudes of strongly dominant harmonics, such as annual sea level variations were replicated by the TG and SA measurements. The other estimated amplitudes estimates differ because TG samples sea level variations at a locality whereas SA is an averaged value of sea level variations over an area $\left(0.75^{\circ} \times 0.75^{\circ}\right.$ grid $)$ in the vicinity of the TG station and they are the final product of several SA missions.

The difference of the two velocity estimates calculated using SA and TG measurements quantifies the rate of VCM at the TG station to be $-0.66 \pm 0.63 /$ yr. Although the magnitude of the estimated VCM rate is large so is its SE, which suggests that the null-hypothesis $H_{0}: V C M_{S A}-V C M_{T G}=$ 0 cannot be rejected. However, GPS measurements reveal statistically significant VCM within the vicinity of the TG station $(-1.31 \pm 0.14 / \mathrm{yr})$. Note that because the GPS station is not colocated at the TG station (16 km away), they may experience different local subsidence, which are unknown to us.

One of the several reasons for the TG and SA measurements failing to detect a statistically significant VCM includes the difficulty of modelling low frequency sea level variations for short SA records as evidenced in Table 1. Another reason is the omission of the correlation between the estimates in single model solutions, which may inflate the error of the estimated VCM rate. In the following section, these deficiencies will be ameliorated by conflating SA measurements and TG measurements with long records under a single model-the confluence model.

\footnotetext{
1 We used $95 \%$ confidence level throughout the study.

2 VIFs are calculated by regressing each one the parameter over the others.
} 
Table 1. Statistically significant $(95 \% \mathrm{CL}) \mathrm{SA}$ and TG solution parameters. Trend and acceleration estimates and their $1 \sigma$ standard errors (SE) are in $\mathrm{mm} / \mathrm{yr}$ and $\mathrm{mm} / \mathrm{yr}^{2}$. The amplitudes of the periodicities (yr) and their SE are in mm. NA: Not Available.

\begin{tabular}{lllll}
\hline \multirow{2}{*}{ Parameter } & Amplitude & SE & Amplitude & SE \\
\cline { 2 - 5 } & \multicolumn{1}{c}{ TG } & \multicolumn{3}{c}{ SA } \\
\hline \hline Trend & 3.96 & 0.09 & 3.31 & 0.61 \\
Acceleration & 0.016 & 0.005 & NA & NA \\
75 & 13.71 & 5.54 & NA & NA \\
56 & 19.10 & 5.68 & NA & NA \\
22 & 9.77 & 3.03 & 17.69 & 6.37 \\
18.6 & 10.16 & 4.17 & 13.34 & 4.69 \\
12.4 & 15.02 & 2.93 & NA & NA \\
11 & 8.09 & 2.84 & NA & NA \\
9 & NA & NA & 10.61 & 4.93 \\
5 & 10.00 & 2.85 & NA & NA \\
3 & NA & NA & 10.38 & 4.69 \\
2.6 & 8.64 & 2.84 & NA & NA \\
2 & NA & NA & 12.73 & 4.61 \\
Chandler & 8.12 & 2.76 & NA & NA \\
Annual & 64.38 & 3.86 & 65.38 & 5.83 \\
Semi Annual & 27.73 & 3.51 & NA & NA \\
Trimestral & NA & NA & 4.93 & 2.25 \\
\hline
\end{tabular}

\subsection{The confluence model}

Stacking the two models for the SA and TG records using the generic equation given by eq. (1) should be the next step for solving periodicities, absolute and relative sea level trend parameters. Nonetheless, such an approach fails to resolve the issues discussed in the previous section. Because SA records are short and located at the end of the TG time series, they unduly influence the estimates, an effect known as, data with high leverage (Belsley et al., 1980). Moreover, such an approach would still require estimation of the VCM as another step. Alternatively, one can difference SA and truncated TG series, thereby eliminating common periodic sea level variations. But such an approach still fails because SA and truncated TG series are not sufficiently long to account for the effect of low frequency sea level changes. Another approach demonstrated by Iz et al. (2019) successfully estimates VCM using SA and TG measurements via "Condition equations with unknown parameters." Nonetheless this approach will require another step to calculate absolute and relative sea level trends through variance propagation with full V/C matrices. The following kinematics conflation model resolves both problems by modelling the relative linear sea level trend in TG record as a function of SA trend and VCM trend, and the geocentric SA sea level trend as a function of TG trend and VCM trend in SA records,

$$
\begin{aligned}
h_{t}^{T G}= & h_{t_{0}}^{T G}+v^{V C M}\left(t^{T G}-t_{0}^{T G}\right)+v^{S A}\left(t^{T G}-t_{0}^{T G}\right) \\
& +a \frac{1}{2}\left(t^{T G}-t_{0}^{T G}\right)^{2}+\sum_{k=0}^{m}\left\{\alpha_{k} \sin \left[\left(\frac{2 \pi}{P_{k}}\right)\left(t^{T G}-t_{0}^{T G}\right)\right]\right. \\
& \left.+y_{k} \cos \left[\left(\frac{2 \pi}{P_{k}}\right)\left(t^{T G}-t_{0}^{T G}\right)\right]\right\}+\varepsilon_{t}^{T G} \\
h_{t}^{S A}= & h_{t_{0}}^{S A}-v^{V C M}\left(t^{S A}-t_{0}^{T G}\right)+v^{T G}\left(t^{S A}-t_{0}^{T G}\right) \\
& +a \frac{1}{2}\left(t^{S A}-t_{0}^{T G}\right)^{2}+\sum_{k=0}^{m}\left\{\alpha_{k} \sin \left[\left(\frac{2 \pi}{P_{k}}\right)\left(t^{S A}-t_{0}^{T G}\right)\right]\right. \\
& \left.+y_{k} \cos \left[\left(\frac{2 \pi}{P_{k}}\right)\left(t^{S A}-t_{0}^{T G}\right)\right]\right\}+\varepsilon_{t}^{S A} \\
\varepsilon_{t}^{T G} & =\rho_{T G} \varepsilon_{t-1}^{T G}+u_{t}^{T G} 0 \leq\left|\rho_{T G}\right|<1 u_{t}^{T G} \sim \operatorname{iid}\left(0, \sigma_{u^{T G}}^{2}\right) \\
\varepsilon_{t}^{S A} & =\rho_{S A} \varepsilon_{t-1}^{S A}+u_{t}^{S A} 0 \leq\left|\rho_{S A}\right|<1 u_{t}^{S A} \sim \operatorname{iid}\left(0, \sigma_{u^{s A}}^{2}\right)
\end{aligned}
$$

In the above formulations, the relative and absolute (geocentric) velocities are linked to each other through the following constraint,

$$
v^{V C M}+v^{T G}-v^{S A}=0
$$

We will outline the Least Squares Solution, LSS, to be used in estimating the model parameters in the following section.

\section{Restricted least squares}

The observation equations discussed in the previous section can be arranged in the following matrix/vector formats,

$$
\begin{gathered}
\mathbf{y}_{T G}=\mathbf{A}_{T G} \mathbf{x}^{C O N F}+\epsilon_{T G} \\
\mathbf{y}_{S A}=\mathbf{A}_{S A} \mathbf{x}^{C O N F}+\epsilon_{S A} \\
\mathbf{c x}^{C O N F}=\mathbf{0}
\end{gathered}
$$

In these expressions, $\mathbf{c}$ is the $1 \times u$ constraint vector arranged by considering eq. (8) and the coefficients of the harmonics in eq. (4) and eq.(5) where $u$ is the total number of unknown parameters in the vector $\mathbf{x}^{\text {CONF }}$ and $n_{T G}, n_{S A}$ are the number of records for the TG and SA 
measurements respectively. The vectors $\mathbf{y}_{T G}$, and $\mathbf{y}_{S A}$ refer to the corresponding observations with design matrices $\mathbf{A}_{T G}$, and $\mathbf{A}_{S A}$. Denoting,

$$
\begin{aligned}
& \mathbf{y}=\mathbf{A x}^{C O N F}+\varepsilon \quad \mathbf{y}:=\left[\begin{array}{l}
\mathbf{y}_{T G} \\
\mathbf{y}_{S A}
\end{array}\right] \quad \varepsilon:=\left[\begin{array}{l}
\varepsilon_{T G} \\
\varepsilon_{S A}
\end{array}\right] \\
& \mathbf{A}:=\left[\begin{array}{l}
\mathbf{A}^{T G} \\
\mathbf{A}^{S A}
\end{array}\right]
\end{aligned}
$$

we observe that the design matrix $\mathbf{A}$ is not full column rank because the two trends that appear in each observation equation based on eq.(1) - eq.(3) are collinear. But augmenting A with the constraint vector $\mathbf{c}$, removes the rank deficiency ${ }^{3}$,

$$
\operatorname{rank}=(\mathbf{A})=i-1, \quad \operatorname{rank}=\left(\begin{array}{l}
\mathbf{A} \\
\mathbf{c}
\end{array}\right)=u
$$

As before, both records are autocorrelated as discussed before,

$$
\begin{aligned}
\varepsilon^{T G} t_{T G} & =\rho^{T G} \varepsilon^{T G} t_{t_{T G}-1}+u^{T G} t_{T G} \\
0 & \leq\left|\rho^{T G}\right|<1 \quad t_{T G}=1 \ldots n_{T G} \\
\varepsilon^{S A} t_{t_{A A}} & =\rho^{S A} \varepsilon^{S A} t_{t_{S A}-1}+u^{S A} t_{t_{S A}} \\
0 & \leq\left|\rho^{S A}\right|<1 \quad t_{S A}=1 \ldots n_{S A}
\end{aligned}
$$

The corresponding variance/covariance, $\mathrm{V} / \mathrm{C}$, matrix of the autocorrelated disturbances for any one of the time series can be written as (İz and Chen, 1999, Kendall, 1968),

$$
\Sigma=\sigma^{2} \cdot\left[\begin{array}{ccccc}
1 & \rho & \rho^{2} & \cdots & \rho^{n-1} \\
\rho & 1 & \cdots & \cdots & \rho^{n-2} \\
\vdots & \vdots & \vdots & \ddots & \vdots \\
\rho^{n-1} & \rho^{n-2} & \rho^{n-3} & \cdots & 1
\end{array}\right]=\sigma^{2} \cdot \mathbf{P}^{-1}
$$

where $\mathbf{P}$ is the corresponding $n_{T G} \times n_{T G}$ or $n_{S A} \times n_{S A}$ weight matrix depending upon which records are in consideration and $\sigma^{2}$ is the variance of the disturbances, $\varepsilon$. Implicit in this expression is the assumption that measurements are equally spaced in time. The correlation decreases for increasing time lag because $|\rho|<1$. The above patterned V/C

3 One of the reviewers identified this requirement as orthogonal bordering discussed in Bjerhammar (1973, Chap. 9), which requires that the row vector $\mathrm{c}$ in the constraint to be orthogonal to all rows in matrix A to increase the rank from $u$ - 1 to $u$ for the exact solution to hold. It may also be possible to eliminate one of the trend parameters from the observation equations and calculate the eliminated parameter using adjusted estimates via full $\mathrm{V} / \mathrm{C}$ propagation in a two-steps procedure. This approach however created instability in inverting the resulting normal equations. matrix has an analytical inverse, and is given by (ibid.),

$$
\begin{aligned}
\Sigma^{-1} & =\frac{\sigma^{-2}}{1-\rho^{2}} \mathbf{P} \\
& =\left[\begin{array}{cccccc}
1 & -\rho & 0 & \cdots & 0 & 0 \\
-\rho & 1+\rho^{2} & -\rho & \cdots & 0 & 0 \\
0 & -\rho & 1+\rho^{2} & \cdots & 0 & 0 \\
\vdots & \vdots & \vdots & \ddots & \vdots & \vdots \\
0 & 0 & 0 & \cdots & 1+\rho^{2} & -\rho \\
0 & 0 & 0 & \cdots & -\rho & 1
\end{array}\right]
\end{aligned}
$$

Assuming SA and TG measurements are uncorrelated, we obtain,

$$
\Sigma^{-1}=\left[\begin{array}{cc}
\frac{\sigma_{T G}^{-2}}{1-\rho_{T G}^{2}} \mathbf{P}_{T G} & \mathbf{0} \\
\mathbf{0} & \frac{\sigma_{S A}^{-2}}{1-\rho_{S A}{ }^{2}} \mathbf{P}_{S A}
\end{array}\right]
$$

At this point another difficulty arises because the solution of the corresponding normal equations involves an inversion without the constraint (Uotila, 1988), which is not possible. Fortunately, Toutenburg (1982, pgs. 33-36) offers the following alternative formulation ${ }^{4}$,

$$
\begin{aligned}
& \hat{\mathbf{x}}^{\mathrm{CONF}}=\left(\mathbf{A}_{T G}{ }^{T} \mathbf{P}_{T G} \mathbf{A}_{T G}+\mathbf{A}_{S A}{ }^{\mathbf{T}} \mathbf{P}_{S A} \mathbf{A}_{S A}+\mathbf{c}^{T} \mathbf{c}\right)^{-1} \\
& \left(\mathbf{A}_{T G}{ }^{T} \mathbf{P}_{T G} \mathbf{y}_{T G}+\mathbf{A}_{S A}{ }^{T} \mathbf{P}_{S A} \mathbf{y}_{S A}\right) \\
& \Sigma_{\hat{\mathbf{x}} \text { CONF }}=\hat{\sigma}_{0}^{2}\left(\mathbf{A}_{T G}{ }^{T} \mathbf{P}_{T G} \mathbf{A}_{T G}+\mathbf{A}_{S A}{ }^{\mathbf{T}} \mathbf{P}_{S A} \mathbf{A}_{S A}+\mathbf{c}^{T} \mathbf{c}\right)^{-1} \\
& \left(\mathbf{A}_{T G}{ }^{T} \mathbf{P}_{T G} \mathbf{A}_{T G}+\mathbf{A}_{S A}{ }^{\mathbf{T}} \mathbf{P}_{S A} \mathbf{A}_{S A}\right) \\
& \left(\mathbf{A}_{T G}{ }^{T} \mathbf{P}_{T G} \mathbf{A}_{T G}+\mathbf{A}_{S A}{ }^{\mathbf{T}} \mathbf{P}_{S A} \mathbf{A}_{S A}+\mathbf{c}^{T} \mathbf{c}\right)^{-1} \\
& \hat{\sigma}_{0}^{2}=\frac{\hat{\varepsilon}^{\mathrm{T}} \mathbf{P} \hat{\varepsilon}}{n^{S A}+n^{T G}-u-1}
\end{aligned}
$$

In this expression, the residuals are calculated through the following expression,

$$
\hat{\varepsilon}=\mathbf{y}-\mathbf{A} \hat{\mathbf{x}}^{C O N F}
$$

We used this formulation to estimate the model parameters for the Annapolis TG station using TG and SA records in the following section.

\section{Solution}

The solution residuals for the SA and TG records are displayed in Figure 3. The circular clusters are indicative of

4 Note that this is derivation is not an exact replica of the derivation given in Toutenburg (1982) but a variant of it derived by splitting the design matrix $\mathbf{A}$ in two parts $\mathbf{A}_{T G}$ and $\mathbf{A}_{S A}$, and assuming that the SA and TG measurements are not correlated. 

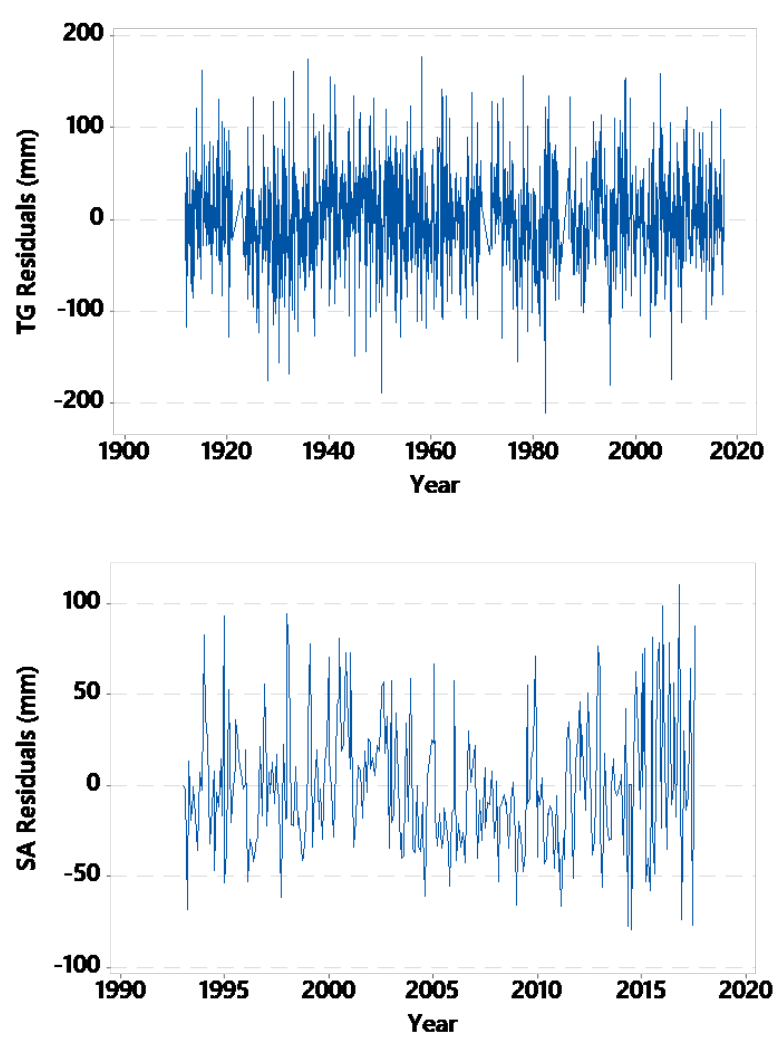

Fig. 3. TG and SA solution residuals calculated using the conflation model.

random variations (Figure 4). Theoretically, the expected value of the Durbin-Watson (DW) statistic is 2 for random distributions. In this solution the calculated DW $=2.00$ for the SA residuals, and DW $=1.95$ for the TG residuals. The estimated parameters for the absolute and relative trends and VCM rates are listed in the third row of Table 2. All the trend estimates are statistically significant. All three estimated linear velocities for absolute and relative sea levels and VCM are consistent in relation to each other and they are statistically well defined. The estimated sea level acceleration coon to the SA and TG measurements in the conflation model is statistically significant, which is not the case when it is estimated from SA records only. More importantly, the null hypothesis for the estimated VCM rate: $H_{0}: V C M_{S A}-V C M_{T G}=0$ is now rejected, i.e., the linear rate of VCM is statistically significant. Although the magnitude of the estimated rate of the VCM is almost $50 \%$ smaller than the one estimated from GPS measurements, the VCM trend estimate based on the conflation model is likely to be more reliable because it is based on 24 years of data span as compared to the VCM trend estimate using 9 years of GPS records. Again as noted before, because the GPS station is not colocated at the TG station (16 km away),
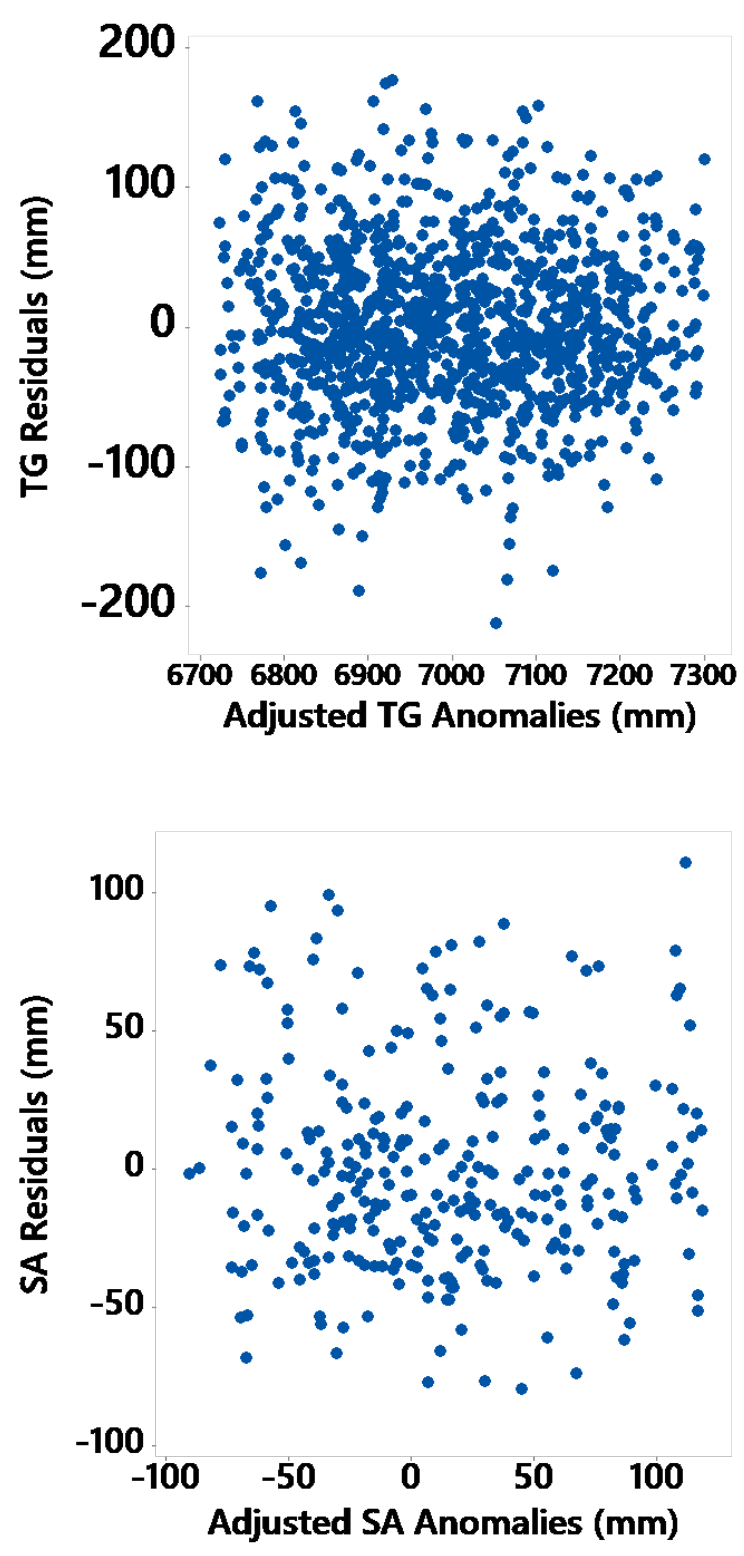

Fig. 4. Atlantic City TG and SA solution residuals plotted against their adjusted records. Circular clusters are indicative of randomly distributed residuals.

they may experience different local subsidence, which are unknown to us.

\section{Conclusion}

In this study, we demonstrated that SA and sufficiently long TG records can be used effectively for all TG stations at coastal areas in quantifying absolute and relative sea level trends and acceleration, together with linear rate of 
Table 2. Estimated trends and acceleration. N/S: Not Significant at $95 \% \mathrm{CL}$. SE of the estimates are $1 \sigma$.

\begin{tabular}{|c|c|c|c|c|c|c|}
\hline $\begin{array}{l}\text { Parameter } \\
\text { /Model }\end{array}$ & Velocity SA /yr & Velocity TG /yr & $\begin{array}{l}\text { Acceleration } \\
\mathrm{SA} / \mathrm{yr}^{2}\end{array}$ & Acceleration $\mathrm{TG} / \mathrm{yr}^{2}$ & $\mathrm{VCM} / \mathrm{yr}$ & VCM GPS /yr \\
\hline Single & $3.30 \pm 0.62$ & $3.96 \pm 0.09$ & $\mathbf{N} / \mathbf{S}$ & $0.016 \pm 0.005$ & $-0.66 \pm 0.63$ & $-1.31 \pm 0.14$ \\
\hline Conflated & $2.71 \pm 0.52$ & $3.44 \pm 0.27$ & $0.015 \pm 0.006$ & $0.015 \pm 0.006$ & $-0.74 \pm 0.26$ & $-1.31 \pm 0.14$ \\
\hline
\end{tabular}

change in VCMs accurately. It is not always evident that SA will provide better results for estimating VCM than GNSS when combined with TG. However, if the local SA measurements are reliable, this approach will provide better estimates for the linear rate of changes in VCMs at TG stations at coastal areas around the globe than those that would be obtained from GPS measurements because such evaluations will always be based on longer SA records than those based on GPS records. This outcome, however, does not mean that GPS measurements are redundant. SA is not a replacement for a co-located assessment. GPS will always be needed as consilience values or can be incorporated into the conflation model as additional information. It is also possible to incorporate GPS measurements if available into the demonstrated kinematic and statistical models as prior information about VCM once they are verified to be compatible with the indirectly available VCM information via SA.

Acknowledgments: We thank an anonymous reviewer for meticulous reading and commenting that improved the manuscript.

\section{References}

Belsley, D.A., Kuh, E., and Welsh, R.E., 1980, Regression Diagnostics: Identifying Influential Data and Sources of Collinearity, John Wiley, New York.

Berrisford, P., Kållberg, P., Kobayashi, S., Dee, D., Uppala, S., Sions, A.J., Poli, P. and Sato, H., 2011, Atmospheric conservation properties in ERA-Interim, Quarterly Journal of the Royal Meteorological Society, 137(659), pp.1381-1399.

Bjerhammer, A., 1973, Theory of errors and generalized matrix inverses, Elsevier Scientific Publishing Co., Amsterdam.

Compo, G.P., Whitaker, J.S. and Sardeshmukh, P.D., 2006, Feasibility of a 100-year reanalysis using only surface pressure data, Bulletin of the American Meteorological Society, 87(2), pp.175190.

Dee, D.P., Uppala, S.M., Sions, A.J., Berrisford, P., Poli, P., Kobayashi, S., Andrae, U., Balmaseda, M.A., Balsamo, G., Bauer, D.P. and Bechtold, P., 2011, The ERA-Interim reanalysis: Configuration and performance of the data assimilation system. Quarterly Journal of the royal meteorological society, 137(656), pp.553-597.

Fadil, A., P. Denys, R. Tenzer, H. R. Grenfell, and P. Willis 2013, New Zealand 20th century sea level rise: Resolving the vertical land motion using space geodetic and geological data, J. Geophys. Res. Oceans, 118, 6076-6091.

Hildreth, G. and Lu T., 1960, Demand relations with autocorrelated disturbances, Technical Bulletin 276, Michigan State University Agricultural Experiment.

İz H.B., T.Y. Yang, C.K. Shum, C.Y. Kuo, 2019, Optimal mathematical and statistical models to estimate vertical crustal movements using satellite altimetry and tide gauge data, J. Geod. Sci., pp. 144-153.

İz H.B., C.K. Shum, C. Zhang, C.Y. Kuo, 201, Inferring Regional Vertical Crustal Velocities from Averaged Relative Sea Level Velocities, J. Geod. Sci., Vol. 7, pp. 59-67.

İz H.B., 2015, More confounders at global and decadal scales in detecting recent sea level accelerations, J. of Geod. Sci, 5, 192-198.

İ H.B., 2014: Sub and Super Harmonics of the Lunar Nodal Tides and the Solar Radiative Forcing in Global Sea Level Changes, J. Geod. Sci., Vol. 4, pp. 150-165.

İz H.B. and Y. Chen, 1999: VLBI Rates with First Order Autoregressive Disturbances, Journal of Geodynamics, Vol. 28, No. 2-3, pp. 131-145.

Kendall, M.G, 1968, The Advanced Theory of Statistics, Vol. 3, Design and Analysis and Time-Series, Edward Arnold Halsted Press, 4th edition.

Permanent Service for Mean Sea Level (PSMSL), 2018, Tide Gauge Data, Retrieved 24 Oct 2018 from http://www.psmsl.org/data/obtaining/.

Toutenburg, H., 1982, Prior information in linear models, John Wiley and Sons, New York.

Uotila, U., 1988, Analysis of Observations. Lecture Notes, The Ohio State University.

Zlotnicki, Victor; Qu, Zheng; Willis, Joshua., 2016, JPL MEaSUREs Gridded Sea Surface Height Anomalies Version 1609, Ver. 1609, PO.DAAC, CA, USA. At http://dx.doi.org/10.5067/SLREFCDRV1 and Integrated Multi-Mission Ocean Altimeter Data for Climate Research TOPEX/Poseidon, Jason-1, 2, \& 3 User's Handbook Version 4.2., 2019, https://sealevel.nasa.gov/data/tools. Dataset accessed [201901]. 